\title{
Nanomaterial Properties: Size and Shape Dependencies
}

\author{
Grégory Guisbiers, ${ }^{1}$ Sergio Mejía-Rosales, ${ }^{2}$ and Francis Leonard Deepak ${ }^{3}$ \\ ${ }^{1}$ Research Institute for Materials Science and Engineering, University of Mons, Place du Parc No. 23, 7000 Mons, Belgium \\ ${ }^{2}$ Facultad de Ciencias Físico-Matemáticas, Universidad Autónoma de Nuevo León, San Nicolás de los Garza, NL 66450, Mexico \\ ${ }^{3}$ The International Iberian Nanotechnology Laboratory (INL), Av. Mestre José Veiga s/n, Braga 4715-330, Portugal \\ Correspondence should be addressed to Grégory Guisbiers, gregory.guisbiers@physics.org
}

Received 6 December 2012; Accepted 6 December 2012

Copyright (c) 2012 Grégory Guisbiers et al. This is an open access article distributed under the Creative Commons Attribution License, which permits unrestricted use, distribution, and reproduction in any medium, provided the original work is properly cited.

Nanoscience and nanotechnology are among the most widely used terms in the modern scientific and technological literature. The idea of nanotechnology appeared for the first time in the famous talk "There is plenty of room at the bottom" given by the physicist Richard Feynman at the American Physical Society meeting at Caltech on December 29, 1959. Feynman described a process by which the ability to manipulate individual atoms and molecules might be developed, using one set of precise tools to build and operate another proportionally smaller set and so on down to the needed scale. In the course of this, he noted, scaling issues would arise from the changing magnitude of various physical phenomena: gravity would become less important whereas surface effects would become increasingly more significant. The term nanotechnology was originally defined by Norio Taniguchi in 1974 as follows: "Nano-technology mainly consists of the processing of separation, consolidation and deformation of materials by one atom or by one molecule." Nanotechnology and nanoscience got started in the early 1980s with two major developments: the advances in computing power and material modeling coupled with significant advances in characterization such as the scanning tunneling microscope (STM) and the atomic force microscope (AFM).

The field of nanoscience and nanotechnology is now growing very rapidly. According to the UK Royal Society, nanoscience is defined as the study of phenomena and manipulation of materials at atomic, molecular, and macromolecular scales, where properties differ significantly from those at a larger scale. Nanotechnologies are the design, characterization, production, and application of structures, devices, and systems by controlling shape and size at the nanoscale. Nanomaterials cross the boundary between nanoscience and nanotechnologies and link these both areas together. Generally, nanomaterials deal with sizes of 100 nanometers or smaller in at least one dimension. The material properties of nanostructures are different from the bulk due to the high surface area over volume ratio and possible appearance of quantum effects at the nanoscale. The study of size and shape effects on material properties has attracted enormous attention due to their scientific and industrial importance.

It is therefore a great pleasure to edit this special issue. In this rapidly progressing area of nanoscience and nanotechnology, it is always important to highlight the most recent and active areas that are being pursued and highlight them to the scientific community. Thus, this special issue brings to fore several such closely related yet diverse areas that encompass this field and are actively being investigated. The papers that appear in this special issue have been grouped according to their themes: size effect on mechanical properties, size effect on catalytic properties, and so forth.

The effect of size in the structural and electronic properties of quantum dots, using DFT calculations, is the topic of study of the paper "A first-principle study of B- and P-doped silicon quantum dots," by J. Zeng and H. Yu; they found that the structural distortions on the quantum dots depend both on the kind of dopant and on the size of the dots. The paper entitled "Cubic-to-tetragonal phase transitions in Ag-Cu nanorods" by F. Delogu and M. Moscia describes molecular dynamics simulation results concerning the structural phase transition of the $\mathrm{Ag}-\mathrm{Cu}$ nanoalloy. W. W. Zhang et al., also using a computational approach, investigate the size dependence of the resonant frequencies in 
silicon nanowires, and their molecular dynamics simulations show that, at small sizes, surface reconstruction affects greatly the vibrational properties of the nanowires; you can find these results in the paper "Study on vibration behavior of doubly clamped silicon nanowires by molecular dynamics." The paper by C. H. Wong and V. Vijayaraghavan showcases the nanomechanics of single- and double-walled carbon nanotubes using molecular dynamics simulations. Also investigating elastic properties, D. X. Lei take a more analytical approach to study the properties of nanocontacts in the paper "Elastic analysis for nanocontact problem with surface stress effects under shear load." The mechanical behavior of a microcantilever has been investigated by X. Wang and F. Wang in the paper "Size-dependent dynamic behavior of a microcantilever plate." D. Bracho et al. investigate the use of silica nanoparticles in the preparation of nanocomposites in the paper "Functionalization of silica nanoparticles for polypropylene nanocomposite applications," where they report interesting dependencies of the mechanical properties of nanocomposites on the size of silica particles. The size and shape dependencies on the melting temperature of gallium nitride has been discussed by D. Arivuoli and P. Antoniammal while the size and shape dependence of ferromagnetism in nanomagnets has been discussed by Y. Li et al. The paper entitled "Magnetic properties of FePt nanoparticles prepared by sonoelectrodeposition" by N. H. Nam et al. describes the method of sonoelectrodeposition as a facile method to successfully synthesize large quantities of ferromagnetic $\mathrm{Fe}_{45} \mathrm{Pt}_{55}$ nanoparticles. The synthesis and colloidal stability of $\mathrm{Fe}_{3} \mathrm{O}_{4}$ magnetic nanoparticles which can have potential applications for hyperthermia are highlighted in the paper "Effect of tetramethylammonium hydroxide on nucleation, surface modification and growth of magnetic nanoparticles" by A. L. Andeade et al. In the paper "One-step method for preparation of magnetic nanoparticles coated with chitosan," K. M. Gregorio-Jáuregui et al. propose another simple method for the production of magnetic nanoparticles.

The effect of size in the catalytic properties of gold nanoparticles supported on glassy carbon is investigated in the paper "Glassy carbon electrode-supported Au nanoparticles for the glucose electrooxidation: on the role of crystallographic orientation," by M. Guerra-Balcázar et al. The effect of size and shape on the toxicity of gold nanoparticles has been investigated by Y. Zhang et al. The catalytic properties of platinum nanoparticles have been studied by J. R. Applegate et al. in the paper entitled "Catalysis of methanol-air mixture using platinum nanoparticles for microscale combustion." The study done by M. M. Mahlambi et al. shows that $\mathrm{m}-\mathrm{TiO}_{2}$ nanocatalyst has the potential to be used in water treatment processes for the degradation of pollutants using solar energy as the source of irradiation energy.

The review paper "Spark plasma sintering of metals and metal matrix nanocomposites" by N. Saheb et al. describes the spark-plasma-sintering process as an effective method to generate successfully a wide variety of metals, alloys, and metal matrix nanocomposites. The work of J. Koruza et al. on the paper "Top-down processing of $\mathrm{NaNbO}_{3}$ nanopowder" focuses in the establishment of a straightforward method to produce nanopowders with a fair control on the size of the particles. The synthesis of quantum dots has been discussed in the paper "Polycation-capped CdS quantum dots synthesized in reverse microemulsions" by $\mathrm{K}$. Lemke and J. Koetz. The synthesis of $\mathrm{Zn}-\mathrm{ZnO}$ core-shell particles was described and characterized by López et al. The optical properties of silicon nanoparticles embedded in a matrix have been investigated by A. Coyopol et al. The thermoluminescence properties of different diameter $\mathrm{ZnS}$ Nanoparticles are described in the paper entitled "Particle size effect on TL emission of ZnS nanoparticles and determination of its kinetic parameters" by L. R. Singh and S. D. Singh. The production of reactive oxygen species under the light irradiation of two different types of $\mathrm{TiO}_{2}$ nanocrystals has been studied by L. Fruk et al. The paper on biomineralization processes by S. Sprio et al. describes the synthesis of hybrid hydroxyapatite/collagen scaffolds for bone and osteochondral regeneration. Finally, let us present an application using nanoparticles; a passive capacitor sensor using $\mathrm{SnO}_{2}$ nanoparticles is presented by $\mathrm{M}$. Agarwal et al. for the detection of ethylene gas.

As the reader may realize, the papers that conform this issue are connected not only by the nature of the systems that are being investigated, and by the techniques used for synthesis and measurement, but also for the emphasis on size effects on the properties of their subjects of study. We believe that we are living in an exciting age where these size dependencies offer both challenges and opportunities, and that, if we take the appropriate approach, this will give us more room for discoveries and applications, even more than the plenty of room that Feynman was thinking on.

Grégory Guisbiers Sergio Mejía-Rosales Francis Leonard Deepak 

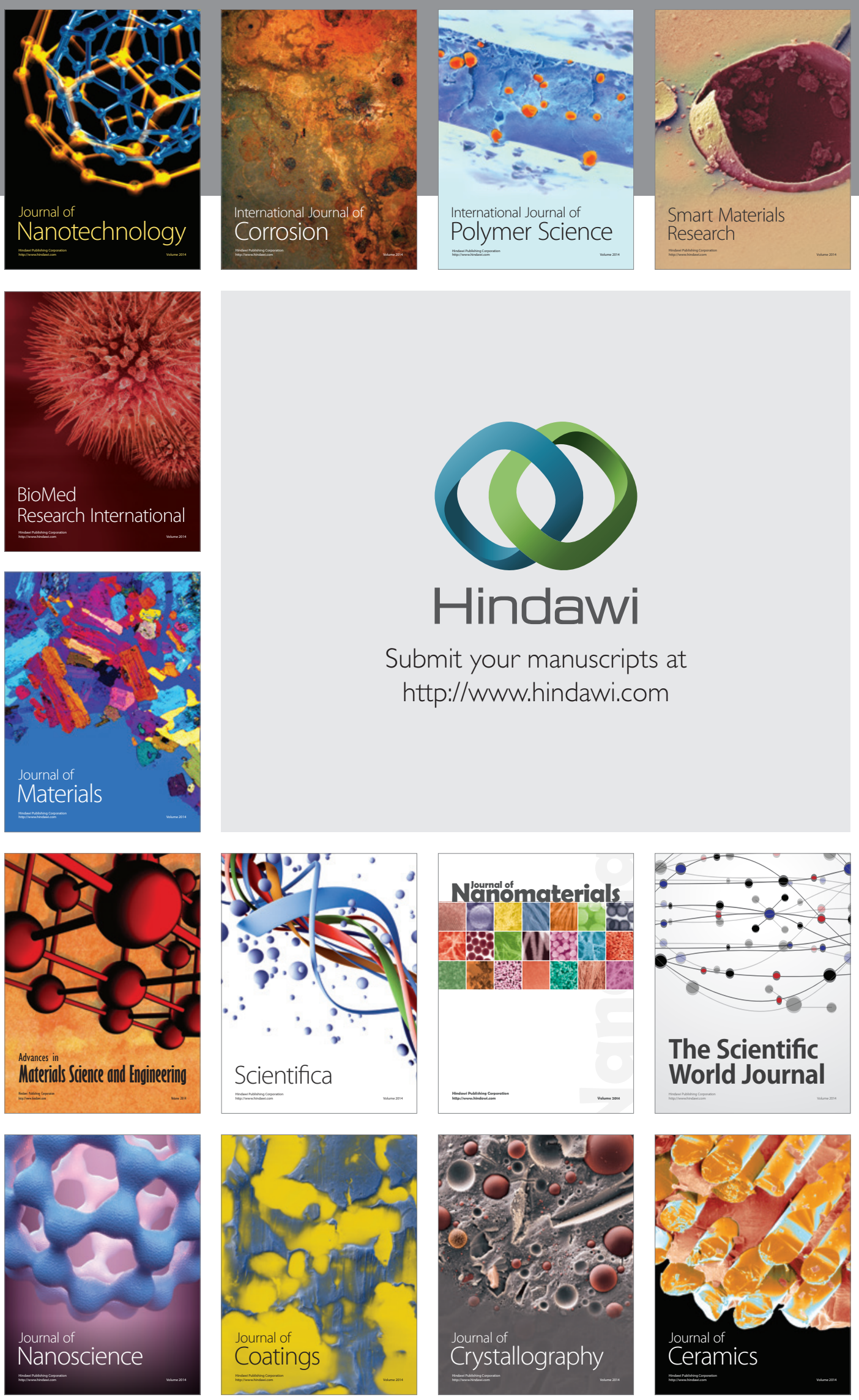

The Scientific World Journal

Submit your manuscripts at

http://www.hindawi.com

\section{World Journal}

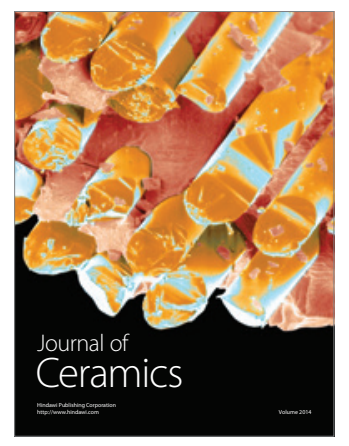

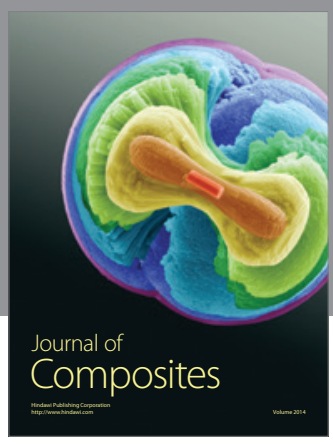
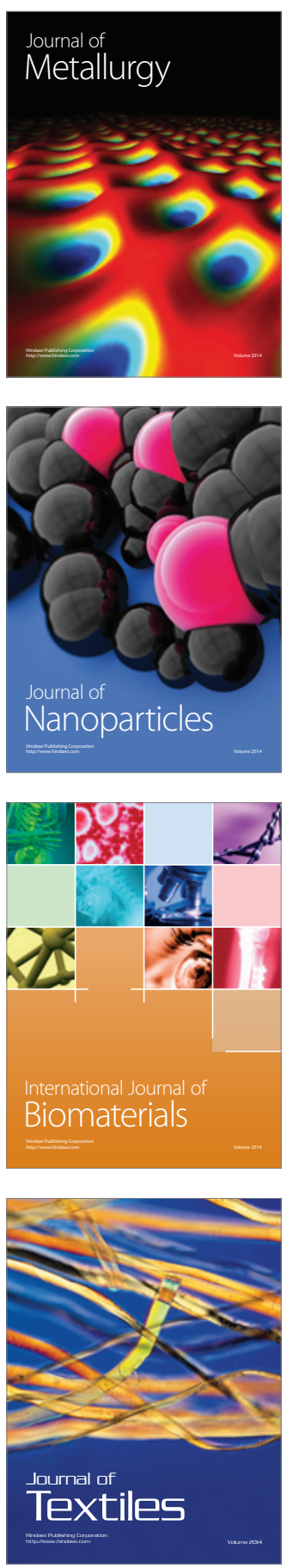